\title{
Egg limitation in host-parasitoid dynamics: an individual-based perspective
}

\author{
Toshinori Okuyama
}

Received: 26 August 2014 / Accepted: 12 January 2015 / Published online: 27 January 2015

(C) Springer Science+Business Media Dordrecht 2015

\begin{abstract}
Theoretical models of parasitoid-host dynamics predict that egg limitation in parasitoids destabilizes community dynamics. However, although egg limitation is experienced by individual parasitoids with variable success of encountering hosts, such details were neglected in previous models. This study developed an individualbased parasitoid-host model that explicitly incorporates egg limitation and host encounters of individual parasitoids. The model indicates that the combination of egg limitation and variation in the success of encountering hosts stabilizes parasitoid-host dynamics. The stabilizing mechanism emerges from Jensen's inequality because egg limitation makes the number of offspring inherently concave down in the number of encountered hosts. Reasons for the inconsistent predictions of the effect of egg limitation between the current model and previous models are discussed.
\end{abstract}

Keywords Persistence · Coexistence · Jensen's inequality $\cdot$ Individual-based model

\section{Introduction}

The numerical response is the change in consumer density as a function of changes in resource density (Solomon 1949), and the reproduction of a consumer is an important component of the numerical response. Models of consumer resource dynamics typically assume that reproduction $\beta$ (e.g., number of offspring in discrete time models) is linearly proportional to the amount of resources acquired

T. Okuyama $(\bowtie)$

Department of Entomology, National Taiwan University

Taipei 106 Taiwan

e-mail: okuyama@ntu.edu.tw
(Murdoch et al. 2003). That is, $\beta=b f$, where $f$ is the functional response describing resource acquisition and $b$ is the parameter describing the consumer's efficiency in converting the acquired resource into offspring. The effects of nonlinear functional response are well studied while assuming $\beta=b f$ (Murdoch et al. 2003). However, the relationship between functional response $f$ and reproduction $\beta$ can be an important driver of population dynamics, and this relationship does not have to be linear, as commonly assumed (Crawley 1975).

In parasitoid-host dynamics, the linear model is also commonly used (e.g., Nicholson and Bailey, 1935; May 1978). In fact, because parasitoids parasitize hosts rather than consume them, occasionally, it is explicitly discussed that complications regarding numerical response are weak to absent in parasitoid-host dynamics (Hassell 1978; May 1978; Murdoch et al. 2003). Nevertheless, empirical evidence for egg limitation in parasitoids exists for both proovigenic and synovigenic species (reviewed in Heimpel and Rosenheim, 1998), and egg limitation is a factor that can create a nonlinear relationship between functional response and reproduction. It is important to understand how egg limitation influences the dynamics of parasitoid-host communities.

There are two modeling approaches to examine the effect of egg limitation on parasitoid-host dynamics (van Baalen 2000). One approach is to include egg limitation as a factor affecting functional response (Getz and Mills 1996). For example, $f$ in $b f$ decreases as egg limitation increases. Thus, egg limitation generally acts in the same manner as the handling time of the functional response. The other approach is to explicitly model an individual's egg limitation independent of functional response such that the relationship between $f$ and $\beta$ is not linear (Shea et al. 1996; van Baalen 2000). These models generally show that egg 
limitation is destabilizing because egg limitation makes parasitoids more inefficient in suppressing hosts as the host density increases (Hassell and May 1973; Hassell 1978).

One of the missing factors in the previous models is the explicit consideration of individual variation. For example, patchy distribution of hosts is a stabilizing factor (May 1978). This mechanism operates because hosts experience unequal pressure of parasitization (e.g., individual variation among hosts). Consequently, parasitoids also experience unequal parasitization success because some individuals encounter more hosts than others. Egg limitation would most strongly act on parasitoids that encounter a large number of hosts. However, the functional response-based modeling (e.g., Getz and Mills 1996) does not incorporate this detail, and it effectively assumes that egg limitation can be averaged among parasitoids. Even when egg limitation was modeled at the individual level (Shea et al. 1996), such variation was neglected. In addition, the model developed by Shea et al. (1996) is a continuous time model that is not directly analogous to the conventional discrete-time parasitoid-host models (further discussed in "Discussion" section). van Baalen (2000) developed a model that considers egg limitation with regard to parasitoid variability, but the focus of the study was the life history of parasitoids rather than the population dynamics.

This study developed an individual-based parasitoid-host model by extending a well-studied model developed by May (1978). In particular, the individual-based model illustrates that egg limitation can stabilize the host-parasitoid community, contrary to previous results. The stabilizing mechanism and the reasons for the inconsistent conclusions are discussed.

\section{The model}

The parasitoid-host model described by May (1978) is considered. Parasitoids exploit hosts that are patchily distributed, and the distribution of hosts is described by a negative binomial distribution. If the number of hosts at time $t$ is $H_{t}$ and the number of parasitoids at time $t$ is $P_{t}$, then the host-parasitoid dynamics can be described as follows:

$$
\begin{aligned}
& H_{t+1}=\quad R H_{t}\left(1+\frac{a P_{t}}{k}\right)^{-k} \\
& P_{t+1}=c H_{t}\left\{1-\left(1+\frac{a P_{t}}{k}\right)^{-k}\right\},
\end{aligned}
$$

where $R$ is the host net rate of increase, $c$ is the number of surviving parasitoids emerging from a parasitized host, $a$ is the per capita attack rate of the parasitoid, and $k$ is the parameter of the negative binomial model describing the distribution of the host. In Eq. 2, $H_{t}\left(1-\left(1+\left(a P_{t}\right) / k\right)^{-k}\right), c$, and $P_{t+1}$ are $f$ (number of hosts acquired), $b$ (reproduction efficiency indicating the number of new parasitoid offspring that can emerge from an acquired host), and $\beta$ (number of new offspring) in the first paragraph of the "Introduction" section, respectively. Thus, Eq. 2 follows $\beta=b f$ discussed earlier although the equation is not per-capita based, and the parameter $c$ would also be influenced by the survival of the parasitoid offspring. The model is stable (e.g., converges to a stable coexistence equilibrium) when $k<1$. When $k>1$, the population fluctuation increases with time and coexistence is impossible (May 1978).

\section{Individual-based model}

The model (Eqs. 1-2) (henceforth referred as the original model) is translated into an individual-based model. For each time step, the total number of encountered hosts will be as follows:

$Q_{t}=\operatorname{nint}\left(H_{t}\left\{1-\left(1+\frac{a P_{t}}{k}\right)^{-k}\right\}\right)$,

where $\operatorname{nint}(x)$ is the nearest integer of $x$. To determine the number of hosts encountered by each parasitoid, a random vector is generated from a dirichlet-multinomial distribution (the subscript for time is omitted for the description of dirichlet-multinomial distribution) (Johnson et al. 1997). The dirichlet-multinomial distribution has the size parameter $Q$ and the dirichlet parameter vector of length $P$ whose elements are all $q$. The value of $q$ influences the variation in number of encounters with hosts (Eq. 4). The random vector is characterized by $\left(U_{1}, U_{2}, \ldots, U_{P}\right)$, where $\sum U_{i}=Q$ and $0 \leq U_{i} \leq Q$. That is, $U_{i}$ is the number of hosts encountered by the $i$ th parasitoid. The distribution is characterized by $E\left(U_{i}\right)=Q / P$ and

$\operatorname{Var}\left(U_{i}\right)=\frac{Q(P-1)(P q+Q)}{P^{2}(P q+1)}$

where $E\left(U_{i}\right)$ and $\operatorname{Var}\left(U_{i}\right)$ are the expected value and the variance of the random variable $U_{i}$, respectively. As $q$ decreases, the variance in the number of hosts encountered increases, and thus, the between-individual variation also increases.

The number of offspring for a parasitoid with $U$ hosts is $\min \left(b_{m}, b_{1} U\right)$, where $b_{1}$ is the number of eggs that can successfully develop from a host and $b_{m}$ is the total number of eggs per parasitoid. For simplicity, all emerged offspring are assumed to survive to the next time step. When there is no limitation (i.e., when $b_{m}=\infty$ ), the individual-based model is basically the same as the original model, except that the densities are assumed to be in discrete numbers. The maximum number of hosts that can be parasitized by a parasitoid is $\left\lceil b_{m} / b_{1}\right\rceil$. Therefore, $u_{i}=\max \left(0, U_{i}-\left\lceil b_{m} / b_{1}\right\rceil\right)$ hosts 
will escape from parasitoid $i$. On the basis of these rules, the host-parasitoid dynamics can be described as follows:

$$
\begin{aligned}
& H_{t+1}=\operatorname{nint}\left\{R\left(H_{t}-Q_{t}+\sum_{i=1}^{P_{t}} u_{i}\right)\right\} \\
& P_{t+1}=\operatorname{nint}\left(\sum_{i=1}^{P_{t}} \min \left(b_{m}, b_{1} U_{i}\right)\right) .
\end{aligned}
$$

The model is analyzed by simulations. The initial number of hosts and parasitoids are $\left(H^{*}+5, P^{*}+5\right)$, where $H^{*}$ and $P^{*}$ are the nearest integers of the equilibrium densities of Eqs. 1-2 when $c=1$ (May 1978). The default parameters used are $a=0.02, R=1.1$, and $b_{1}=1$. The remaining parameters are systematically changed to examine the effect of egg limitation $\left(b_{m}\right)$, individual variation in the success of encountering $(q)$, and the distribution of hosts $(k)$. Coexistence is defined for persistence of 1000 time steps. The probability of coexistence for each parameter combination is quantified using 50 replications.

\section{Results}

First, it is worth noting that, without egg limitation $\left(b_{m}=\right.$ $\infty)$, parasitoid-host dynamics are altered in important ways from those predicted by the original model. When $b_{m}=$ $\infty$, individual variation does not influence the model (see "Discussion" section for explanation), and thus, the effect is solely attributed to the discreteness (i.e., the use of the nearest integer function in the model). The original model displays either stable equilibrium dynamics (when $k<1$ ) or fluctuations that increase with time (when $k>1$ ). The individual-based model can exhibit limit cycle dynamics, and the host-parasitoid dynamics can persist through equilibrium or limit cycles even when $k>1$ (Fig. 1). Although

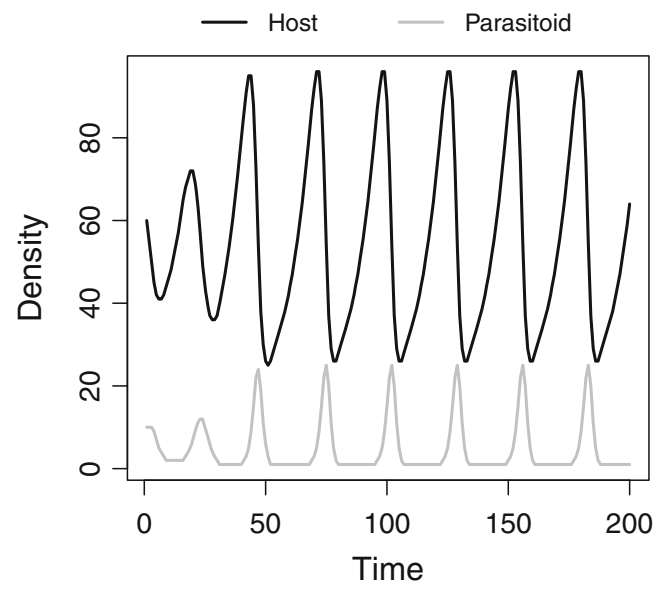

Fig. 1 Population dynamics without egg limitation $\left(b_{m}=\infty\right)$ when densities are discrete and $k=2$. The value of $q$ has no effect in this model discreteness can stabilize host-parasitoid dynamics, arbitrarily increasing $k$ destabilizes the community. Along the default parameters, when $k$ is greater than approximately 2.3, population oscillation becomes too large and extinction takes place.

With egg limitation, the parameter range that allows coexistence expands for large values of $k$ (Fig. 2). Strong egg limitation (i.e., small $b_{m}$ ) and large individual variation (small $q$ ) facilitate coexistence. The qualitative pattern is robust to changes in $R, a$, and $b_{1}$, as well as the initial densities (Fig. 3). A sample dynamics resulting in coexistence is shown in Fig. 4.

\section{Discussion}

Egg limitation can stabilize parasitoid-host dynamics under the biological assumptions made in the current model. Discrepancies between this study and previous studies arise from variable biological assumptions made by each model. To interpret results, it is important to fully understand the relationship between the model structure and the corresponding biological details. In the following sections, the coexistencemechanism of the current model and the differences between the current model and previous models are discussed.

The stabilizing mechanism of the current model is the combination of egg limitation and individual variation in the success of encountering hosts (Okuyama 2013), and it can be described as follows. Consider, there are ten hosts that are encountered by two parasitoids. When there is no egg limitation, a total of ten offspring will be produced (assuming $b_{1}=1$ ). When $b_{m}=5$, ten offspring will also be produced when there is no individual variation in the encounter (i.e., each parasitoid encounters five hosts). However, when there is individual variation, the result is different. For example, when one parasitoid encounters four hosts and the other parasitoid encounters six hosts, the total number of offspring is nine. When the egg limitation exists, the number of egg laid generally is a concave down function of the number of encountered hosts. The same logic works for any other concave down functions such as $y=a x /(b+x)$ where $a$ and $b$ are the parameters of the model; $y$ (average number of eggs laid) is a concave down function of $x$ (number of hosts encountered). It can be generally demonstrated that when the relationship is a concave down function, as assumed in this situation, then variation in encountering hosts will reduce the total number of parasitoid offspring. Thus, the value of $q$ has no effect, as described in "Results" section, when the relationship between the success of encountering hosts and reproduction is linear (e.g., $b_{m}=\infty$ ). This phenomenon is called Jensen's inequality and is discussed elsewhere 
Fig. 2 The effect of egg limitation and individual variation in host encounter on coexistence. The proportion of simulation runs that resulted in coexistence is indicated by the color key. The contour line indicates probability at 0.05

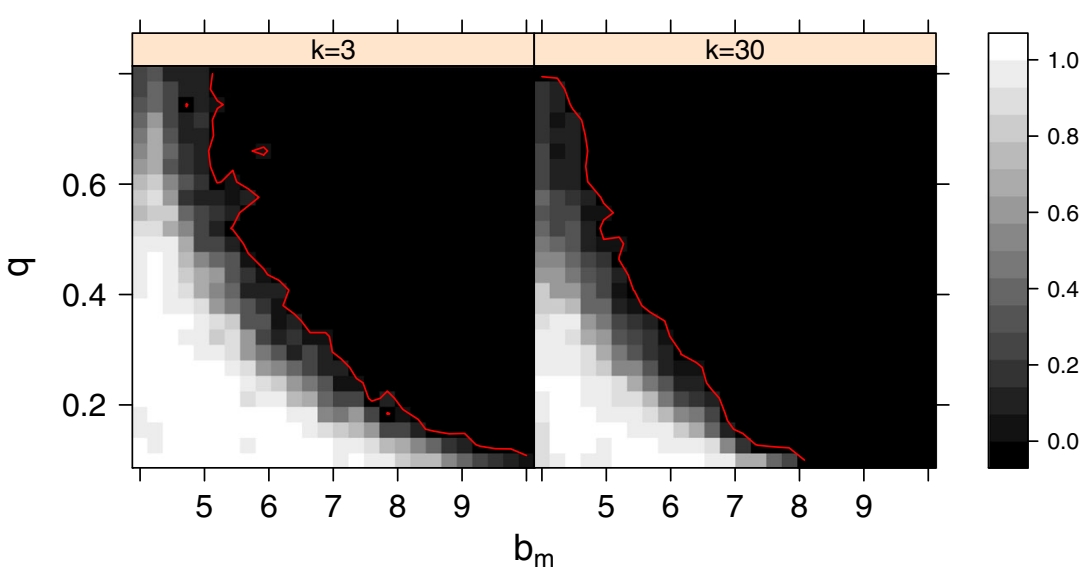

(Ruel and Ayres 1999). However, Jensen's inequality itself is insufficient to stabilize the community.

Some patterns in individual variation can stabilize community dynamics through Jensen's inequality. Consideration of detailed individual variation is important because egg limitation is experienced by individuals that encounter variable numbers of hosts. Getz and Mills (1996) modeled egg limitation through functional response. That is, the proportion of parasitized hosts decreases as host density increases because of egg limitation. Thus, the effect is the same as that of handling time, which is generally thought to destabilize community dynamics (Hastings 1997). However, the issue may be the absence of individual variation and not that egg limitation and handling time are considered equivalent. For example, handling time also produces new dynamics when it is modeled at the individual level (Okuyama 2009; Okuyama 2012).

In consideration of Jensen's inequality, individual variation decreases the number of offspring. Therefore, if the size of variation in host encountering success among parasitoids increases with the number of parasitoids, this creates

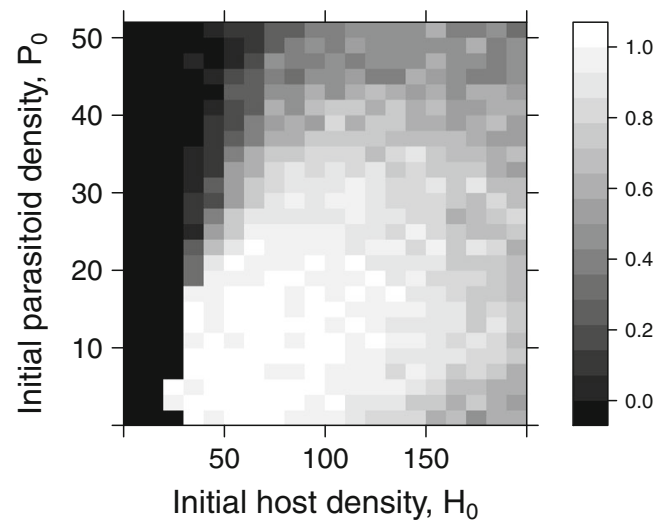

Fig. 3 The effect of the initial host density $H_{0}$ and the initial parasitoid density $P_{0}$ on coexistence. $b_{m}=4.5, q=0.15, k=30$. The initial densities used in Fig. 2 are $H_{0}=60$ and $P_{0}=10$. Coexistence probabilities are estimated based on 50 simulation runs negative density dependence on the parasitoid population, which is a stabilizing factor (Okuyama 2013). Generally speaking, when the fitness of an individual decreases with an increase of the density of conspecifics (e.g., intraspecific competition), it can act as stabilizing force (Hastings 1997). The dirichlet-multinomial distribution assumes this variance pattern (i.e., variance increases with the parasitoid population for a given mean encounter), and it is important to verify the assumption about the distribution of foraging success among parasitoid individuals. For example, if host distribution is not patchy, variation in foraging success among parasitoids may be lower. Host densities may also influence foraging success variation. In other words, although this study assumed $q$ to be constant, it may change with dynamic ecological variables (e.g., $H$ and $P$ ). The parametric assumption itself also requires verification. A difficulty in empirically evaluating these details is that such data (e.g., the success of each parasitoid of encountering hosts) are not available because it is logistically impossible or difficult to obtain data with such details, especially in the field. Methodological advancement that facilitates

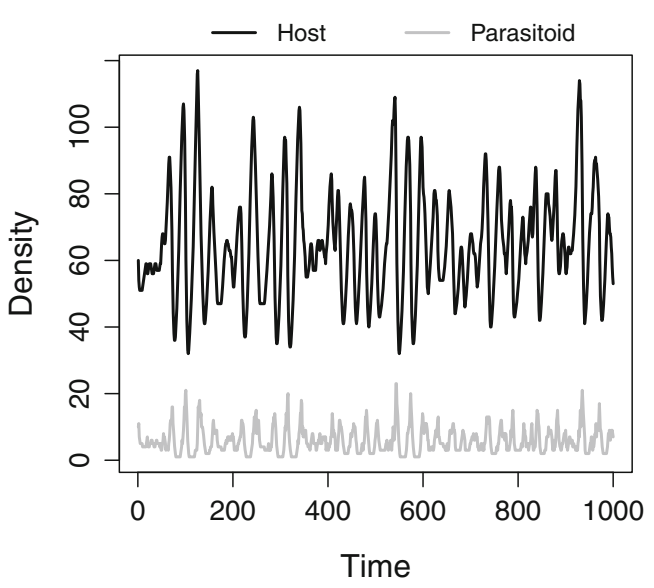

Fig. 4 Population dynamics with egg limitation and individual variation in host encounter. $b_{m}=3, k=10, q=0.5$ 
quantifying oviposition success at the individual level is awaited. Meanwhile, at a simpler level, studies in a simple environment (e.g., laboratory) that allows researchers to track each individual (e.g., video recording or direct observation) can initially provide useful information about factors that affect variation among consumers.

Shea et al. (1996) explicitly modeled the individual's egg limitation using a differential equation model. Because the model is a continuous model, its results cannot be directly compared to those from the common discrete parasitoidhost models. In particular, with regard to egg limitation, the model assumes that a laid egg immediately becomes a new parasitoid and begins laying its own eggs. Thus, although one parasitoid can only lay a limited number of eggs, the model structure effectively eliminates much of the effects of egg limitation. In addition, it is an average-based model, and the effect of individual variation discussed previously is also absent. However, which approach (i.e., continuous vs. discrete) is more appropriate depends on the biological systems and questions.

The assumptions of the current model also need to be carefully interpreted. For example, the model divides $Q$ hosts among $P$ parasitoids for encountering, as if assuming that a host that is encountered by one parasitoid cannot be encountered by another parasitoid. Such an interpretation is mechanistic, but it makes the model unrealistic, and thus, the foraging part of the model needs to be interpreted phenomenologically. After all, the original negative binomial model about the number of parasitized hosts is also a phenomenological approximation (May 1978). Thus, although the model mechanistically incorporates egg limitation, it omits other details. It is possible that detailed interactions among parasitoids when they are searching for hosts can alter the model dynamics. For example, parasitoids change their oviposition behavior as their egg load changes (Minkenberg et al. 1992; Heimpel et al. 1996). Models that mechanistically consider foraging and oviposition would be worthwhile, and they may be necessary for understanding variation among individuals in foraging and oviposition success.

Parasitoid-host models are one of the main components of consumer-resource dynamics (Murdoch et al. 2003), and understanding (de)stabilizing mechanisms is essential for the development of ecological theories. This study reveals that egg limitation, a factor previously considered as destabilizing, is an essential component of a stabilizing mechanism, indicating the importance of individual identity in population and community dynamics. Although the study is motivated from a basic ecological viewpoint, as parasitoids are important biological control agents
(Murdoch et al. 1985; Mills and Getz 1996), examining the current model with a different focus (e.g., effects on pest density) may also elucidate new perspectives in the applications of host-parasitoid dynamics.

Acknowledgments I thank an anonymous reviewer and the handling editor for their comments that improved the manuscript. This study was in part supported by the Ministry of Science and Technology grant number 102-2311-B-002-038-MY3.

\section{References}

Crawley MJ (1975) The numerical responses of insect predators to changes in prey density. J Anim Ecol 44:877-892

Getz WM, Mills NJ (1996) Host-parasitoid coexistence and egglimited encounter rates. Am Nat 148:333-347

Hassell MP (1978) The dynamics of arthropod predator-prey systems. Princeton University Press, Princeton

Hassell MP, May RM (1973) Stability in insect-parasite models. J Anim Ecol 42:693-726

Hastings A (1997) Population biology. Springer, New York

Heimpel GE, Rosenheim JA (1998) Egg limitation in parasitoids: a review of the evidence and a case study. Biol Control 11:160-168

Heimpel GE, Rosenheim JA, Mangel M (1996) Ecology 77:2410 2420

Johnson NL, Kotz S, Balakrishnan N (1997) Discrete multivariate distributions. John Wiley \& Sons, New York

May RM (1978) Host-parasitoid systems in patchy environments: a phenomenological model. J Anim Ecol 47:833-843

Mills NJ, Getz WM (1996) Modelling the biological control of insect pests: a review of host-parasitoid models. Ecol Model 92:121-143

Minkenberg OPJM, Tatar M, Rosenheim JA (1992) Egg load as a major source of variability in insect foraging and oviposition behavior. Oikos 65:134-142

Murdoch WW, Chesson J, Chesson PL (1985) Biological control in theory and practice. Am Nat 125:344-366

Murdoch WW, Briggs CJ, Nisbet RM (2003) Consumer-resource dynamics. Monographs in population biology. Princeton University Press, Princeton

Nicholson AJ, Bailey VA (1935) The balance of animal populationsPart I. Proc Zool Soc Lond 105:551-598

Okuyama T (2009) Local interactions between predators and prey call into question commonly used functional responses. Ecol Model 220:1182-1188

Okuyama T (2012) Behavioral states of predators stabilize predatorprey dynamics. Theor Ecol 5:605-610

Okuyama T (2013) Consequences of variation in foraging success among predators on numerical response. Ecol Evol 3:40394043

Ruel JJ, Ayres MP (1999) Jensen's inequality predicts effects of environmental variation. Trends Ecol Evol 14:361-366

Shea K, Nisbet RM, Murdoch WW, Yoo HS (1996) The effect of egg limitation on stability in insect host-parasitoid population models. J Anim Ecol 65:833-843

Solomon ME (1949) The natural control of animal populations. J Anim Ecol 18:1-35

van Baalen M (2000) Parasitoid population dynamics. In: Hochberg M, Ives AR (eds) The evolution of parasitoid egg load. Princeton University Press, Princeton, pp 103-120 